\title{
The Feasibility of Interactively Probing Quiescent Properties of GUI Applications
}

\author{
Peter Achten \\ Department of Software Technology, Radboud University Nijmegen, Toernooiveld 1, \\ 6525ED Nijmegen, The Netherlands \\ P.Achten@science.ru.nl
}

\begin{abstract}
In this paper we explore how application-users can, in an interactive way, test properties about the state of GUI applications that can be classified as local state transition systems with quiescence. These properties can be added and removed at run-time. It is guaranteed that they are type-correct. We investigate the consequences of such an approach for one particular functional GUI library, Object I/O. The goal is to gain confidence in the quality of interactive applications, and to seek properties that can be proven correct, perhaps using formal proof tools.
\end{abstract}

\section{Introduction}

Programming an effective Graphical User Interface (GUI) is a challenging task because of the myriad of details that need to be controlled and managed: the set of possible events, knowledge of the API, general design rules for GUIs, life-cycle maintenance of GUI objects, and so on.

However, if we ignore this plethora of details, it turns out that the structure of a typical GUI program is basically a nested while-case loop. The while structure reflects the obligation of a GUI application to poll for events until termination; the case structure reflects the need to perform case distinction on the events and act according to the needs of the application; this structure is nested due to the use of constructs such as modal dialogues and synchronous message passing.

A second characteristic feature of GUI applications is that they use a structured state, usually relying on scope rules. This structured state evolves dynamically, as parts of the state are associated with GUI objects. The data itself is in general not very complicated. We call the state stable when the application is polling for the next available event, because it can not modify the state in any way until an event is actually been given to it. In testing theory, this state of the application is also known as quiescence [17], i.e.: the application can not proceed without further input.

Although the structure of GUI programs is clear, it is hard to reason about GUI applications thoroughly and rigidly. This is caused by the following reasons:

1. The actions that are triggered by the case distinctions operate on the same (parts of the) state structure, thereby interfering with each other. When there are many such actions it is hard to keep track of each of their effects. Even a small application such as Notepad on Windows has at least 100 actions. 
2. Reasoning about a particular program run boils down to reasoning about a particular event trace, an ordered sequence of events. Applications modify the set of admissible events dynamically by techniques such as enabling/disabling, hiding/showing, opening/closing of GUI objects, in order to provide the user with proper feedback on allowed actions on his part. This means that one cannot assume that an event trace is a sequence of random events.

3. The case distinction done by applications is partial: a program does not respond to all possible events because that would make even the simplest application unreasonably large. Instead, the underlying system takes standard actions if the application is not interested or chooses to ignore events. As a consequence, one can not rely solely on the code as the specification, but one must also take the behavior of the operating system into account.

When designing the state structure, the programmer usually has some properties in mind that the values of the state structure should satisfy whenever the application is in a stable state. A property is invariant for a specific event trace if it holds during all stable states along this trace. Ideally, we would like to prove that a property is invariant for every possible event trace because this promotes such a property to an invariant of the application. Unfortunately, for the reasons mentioned above, this is infeasible.

In this paper we take a pragmatic approach to the problem of establishing (hopefully invariant) properties of GUI applications. We want to encourage GUI programmers to develop as many properties as possible (including false ones!) to any GUI object of an application under construction or one that has been finished long ago by perhaps somebody else. This is known as run-time assertion checking $[12,10]$. However, for reasons of flexibility, we want to be able to interactively add and remove properties. The application, whenever in a stable state, checks all currently added properties and notifies the user whenever a property is violated. In this way, the developer can probe the application for properties.

The concrete research questions this feasibility study should give an answer to are:

- Can we assign properties to any GUI object in the application, and even to the whole application? Can this be done at compile-time and at run-time?

- Are properties that are added always of the correct type?

- Can we store properties on disk?

- Is there no loss in efficiency when no properties are probed?

Based on the results of this feasibility study, we intend to implement this system for the Object I/O library $[2,4,1,5]$, a comprehensive GUI library that is available for the functional programming languages Clean [16] and Haskell [14].

This paper is structured as follows. We first present our technique for local state transition systems in general in Section 2. In Section 3 we show that Object $\mathrm{I} / \mathrm{O}$ is a local state transition system. We then explain the expected issues when adding property probing to Object I/O in Section 4. We discuss related work in Section 5 and conclude in Section 6. 


\section{Probing Local State Transition Systems}

The bare bones structure of the class of GUI applications that we investigate is that of a local state transition system [3], which is basically the same as that of a nested while-case as discussed in the introduction. In this section we reveal this structure (Section 2.1) in order to point out the technical problems that need to be resolved when adding/removing (Section 2.2) and testing (Section 2.3) properties in a type-safe and dynamic way.

\subsection{Local State Transition Systems}

The set of types is very similar to those presented in [3], except that here we do include interactive processes (in order to reason about complete programs). A program (Program) is a collection of processes (Process), each of which encapsulates a state ps via an existential quantifier ( $\exists$ ps:). This state is shared by all of its elements. It effectively models the global data that is accessible by every element. To enforce this, the type (Proc ps) is used.

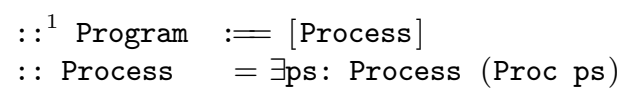

Every process (the record type Proc ps below) has a number of actions that respond to process related events. These are modelled by the list of functions in the field pcbfs. Note that the type of an action, ((Proc ps) $\rightarrow$ (Proc ps)) provides it with full access to all elements of a program. In particular, the other processes are also an element of a process (pcontext). Processes are identified by an ID, which is a simple integer. An event (id,i): : Event identifies the $i$-th action of the process id. This is of course a very simplified form of events.

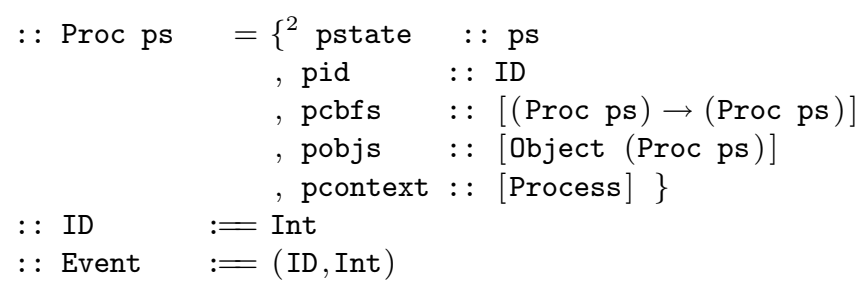

Processes have top-level objects (these correspond with menus, windows, and so on), stored in pobjs, each of which again encapsulate their piece of local state Is and operate on the same state of the program pst, which is always (Proc ps):

$::$ Object pst $=\exists l \mathrm{~s}:$ Object ls [Comp ls pst]

Top-level objects have components with access to the shared state (Proc ps) and the local state of the top-level object (ls). A component (Comp ls pst)

\footnotetext{
${ }^{1}$ All Clean type definitions are introduced by the keyword : : Synonym types are indicated with separator symbol :==. Algebraic and record types are indicated with separator symbol $=$.

${ }^{2}\left\{f_{0}:: t_{0}, \ldots, f_{n}:: t_{n}\right\}$ denotes a record type with field names $f_{i}$ and types $t_{i}$.
} 
is either a concrete object ( $0 \mathrm{bj}$ ls pst), or it replaces the current local state (NewLS 1s pst), or it extends the current local state (AddLS 1s pst).

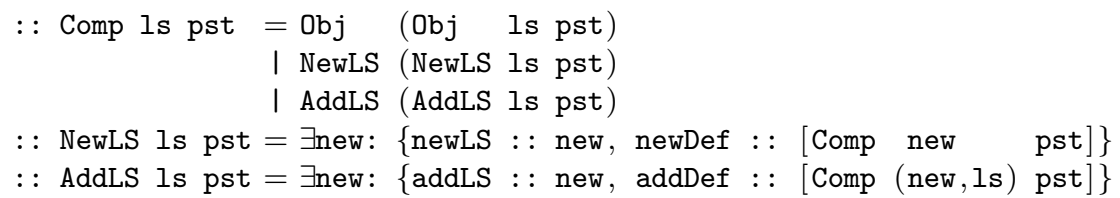

Analogous to processes, concrete objects are identified via an ID, have actions, and can contain other objects. An event (id,i) identifies the i-th action of the concrete object identified by id.

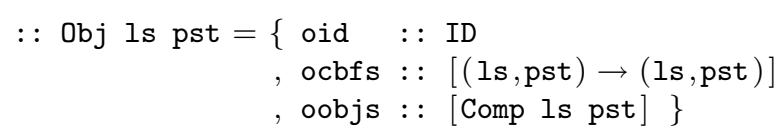

With this collection of types we can model scoped state structures. A value $\mathrm{p}:$ : Program represents the complete quiescent state of a program. When an application successfully polls for an event $e=(i d, i)$, then the next quiescent state of the program is computed by (eval e).

eval : : Event Program $\rightarrow$ Program ${ }^{3}$

We will not discuss its implementation: it is basically a recursive function that searches for a process or concrete object that is identified by id and applies the i-th action to the current program state. Details can be found in [3].

Example In order to make this discussion more concrete, consider the following small example of a local state transition system that has a few 'bugs':

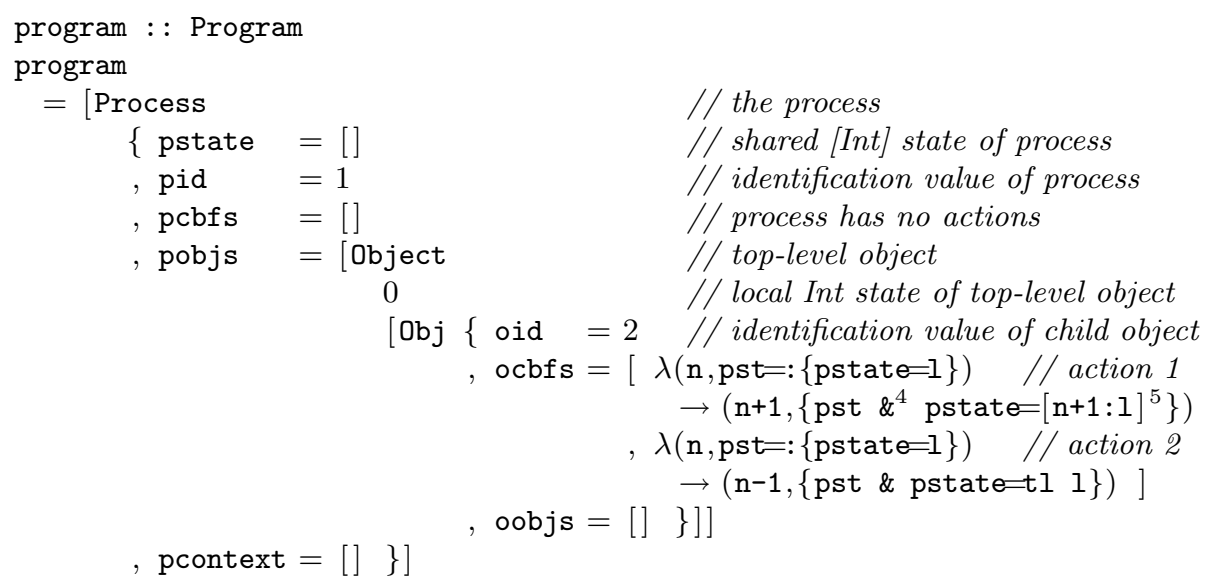

${ }^{3}$ Clean separates function arguments by whitespace, instead of $\rightarrow$.

${ }^{4}\left\{r \& f_{0}=v_{0}, \ldots, f_{n}=v_{n}\right\}$ is a record equal to $r$, except that fields $f_{i}$ have value $v_{i}$.

${ }^{5}$ Clean lists are always delimited by [ and ]. 
The process maintains and shares a list of integers, pstate :: [Int]. The concrete object, identified by oid $=2$, has two actions: the first adds one element to the list, and the second shortens the list. The object has a local integer state which value should reflect the length of the shared integer list. The second action contains two bugs caused by unrestricted uses of tl in tl 1 and - in n-1.

\subsection{Adding and Removing Properties at Run-Time}

A property of some data type st is a boolean function:

$:$ Prop st $:=$ st $\rightarrow$ Bool

In Section 2.1 we have introduced the elements that we want to probe:

- Complete programs, of type [Process] are probed with (Prop [Process]).

- Processes, of type (Proc ps), with ps the type of the shared state, are probed with (Prop (Proc ps)).

- Concrete objects, of type (Obj Is (Proc ps)), with 1s the type of the local shared state of the concrete object. Note that, due to NewLS and AddLS, Is can be a nested tuple composition of several local states. They are probed with (Prop (ls, Proc ps)).

In order to test any of these elements at run-time with an appropriate property one needs to provide a property of the correct type. Unfortunately, only the type of complete programs is immediately accessible; the types of the scoped state of processes and concrete objects can not be retrieved, even though we, as program developer, are well aware of their concrete types. The deliberate existential quantification has rendered it impossible for us to check properties afterwards using a solution within the static type system.

We need to resort to dynamic type checking if we are to solve this issue. For several years now, Clean has had dynamic types $[15,18]$. There are basically two ways to use dynamic types for our problem:

1. Do not use existential types to hide the types of the states but use dynamic types. In that case, checking for type equality is straightforward, using runtime type unification.

2. Use existential types to hide the types of the states, but do the type equality match inside the object's scope where the types are known.

Alternative 1 is alien to the philosophy of working in a strongly typed language. Instead, we show that alternative 2 can be used within the framework.

First we wrap properties in a dynamic, and give such a property a name:

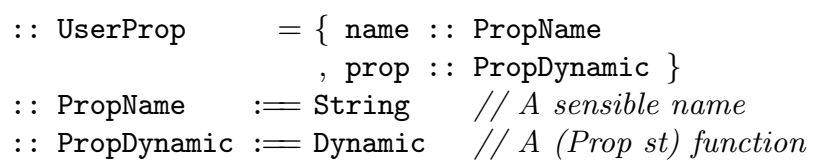


We need to make a few modifications to the data types that we have introduced in Section 2.1. We store the properties of each object in an association list. Its key value is (Just id) with id: : ID of processes and concrete objects, and Nothing for complete programs. The association list is stored globally in the Program type, which now becomes a record.

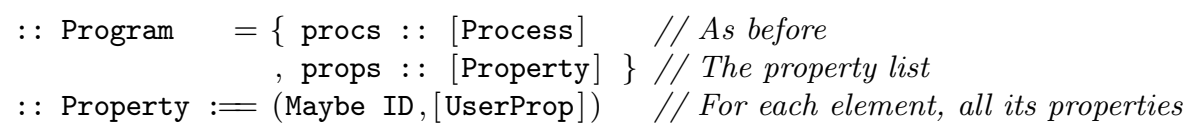

The second change that we need to make is related with the dynamic type system. We are going to match the type of a property encapsulated in a PropDynamic with the state in scope of concrete objects and processes. This is done by a type dependent function [15]. A type dependent function can match a dynamic type with a static type, provided the static type belongs to the TC type class. We need to impose this restriction to the type variables of Proc and Obj. Because Clean does not support type class restrictions on data type definitions, we do this with an explicit dictionary (DictTC) which amounts to the same thing:

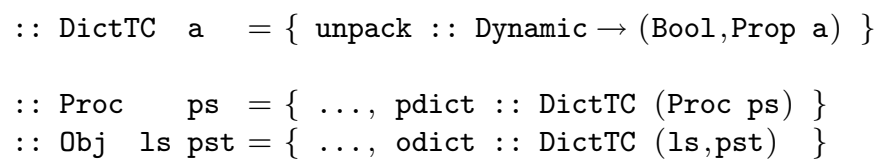

The unpack member is a function that returns the content of its dynamic argument if it correctly contains a property of the right type. It is easy to define a type dependent function that creates a dictionary of the desired type:

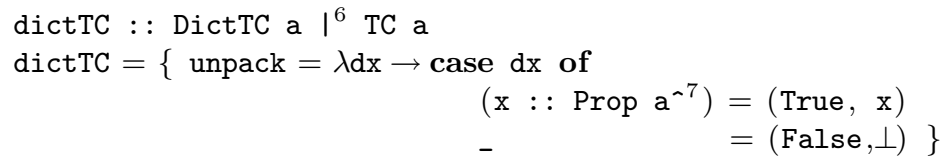

$$
\left(\mathrm{x}:: \text { Prop } \mathrm{a}^{-7}\right)=(\text { True, } \mathrm{x})
$$$$
\text { - } \quad=(\text { False }, \perp)\}
$$

We can now proceed by defining the function addProperty that associates a property with an element:

addProperty :: (Maybe ID) UserProp Program $\rightarrow$ (Bool, Program)

The task of (addProperty mid prop prog) is to extend the prog.props list with an entry for (mid,prop) either by extending an existing entry or creating a new one. The function fails (returns False) if the type of the property does not match. The key challenge of this function is the check for type equality. Let us assume that this function, propertyTypeMatches, exists. Then the definition of addProperty is straightforward:

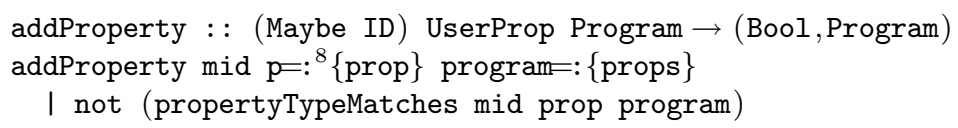

${ }^{6}$ In a function type, | introduces all overloading class restrictions.

${ }^{7} a^{\wedge}$ refers to $a$ in the parent function type, in this case DictTC $a$.

${ }^{8} x=$ : $e$ binds $x$ to $e$. 


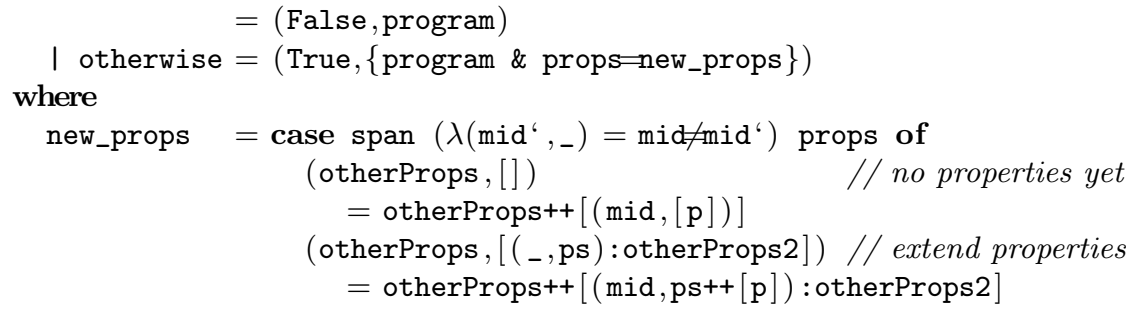

Note that addProperty maintains the order of registered properties, so properties are tested in the same order all the time.

The function application (propertyTypeMatches mid p program) must decide whether the indicated object operates on the type as given by $\mathrm{p}$. If mid $=$ Nothing, then it must be a program property, and hence the dynamic content should be matched with type (Prop [Process]):

propertyTypeMatches :: (Maybe ID) PropDynamic Program $\rightarrow$ Bool

propertyTypeMatches Nothing $d_{0}=$ case dp of

$$
\begin{aligned}
\left(\_ \text {: : Prop }[\text { Process }]\right) & =\text { True } \\
\text { otherwise } & =\text { False }
\end{aligned}
$$

If $($ mid $=($ Just id $))$, then it must either correspond with a process or with a concrete object.

propertyTypeMatches (Just id) dp \{procs $\}=$ any (processMatches id dp) procs

A process matches if its pid matches id and the dynamic property dp matches the dictionary pdict. A process also matches if any of its components matches:

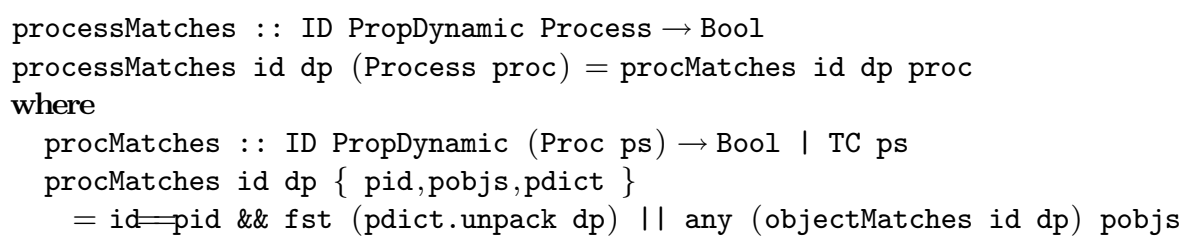

The search for the proper concrete object is handled recursively. The interesting case is the match on a concrete object, which proceeds analogously to matching a process: either the concrete object matches or any of its children.

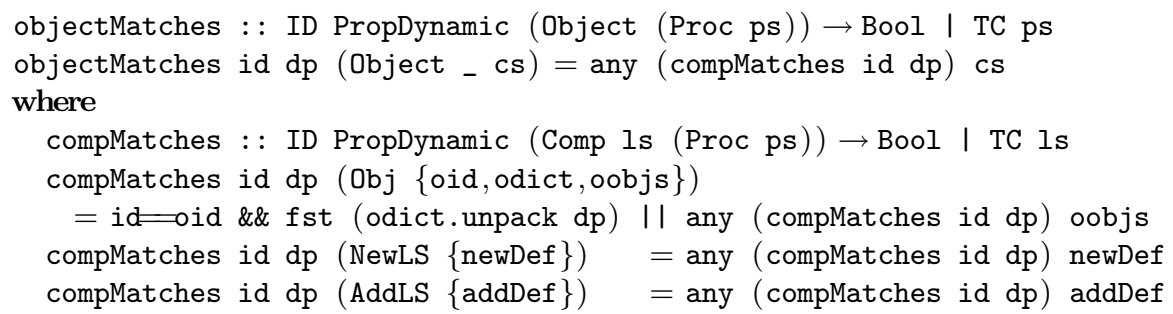

Finally, it is convenient to have a version of addProperty that aborts in case the property type does not match the indicated object's state:

add : : (Maybe ID) UserProp Program $\rightarrow$ Program 
add mid prop program

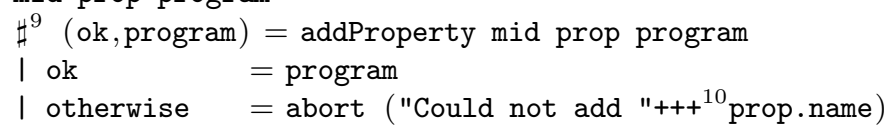

Because properties are stored globally in Program, it is trivial to define the function that removes properties by name:

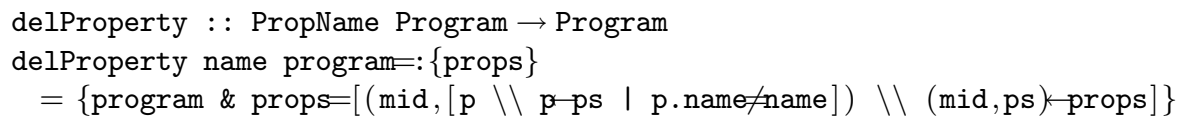

Example We continue our running example given at the end of Section 2.1 by extending it with properties. The only change of definition of program is the extension with two record fields pdict=dictTC and odict=dictTC at the appropriate places, as well as an empty properties list (props $=[]$ ).

We introduce one property for the program and process, and two for the concrete object. They are:

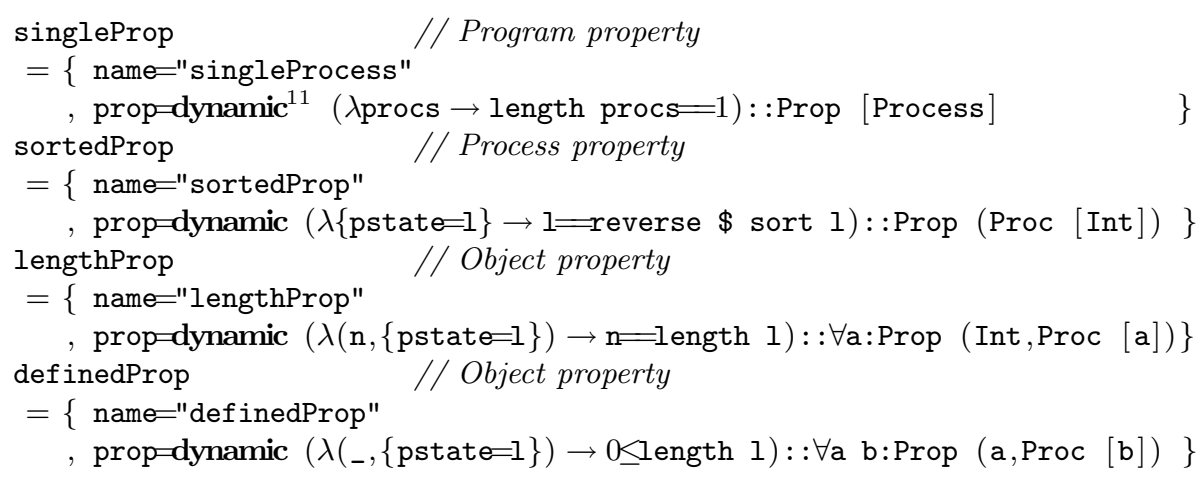

singleProp states that there is one single process at every stable state; sortedProp says that the integer list of the process is in reverse order; lengthProp defines that the integer value of the concrete object correctly keeps track of the length of the list of its parent object; definedProp defines that the list spine does not contain $\perp$. Note that the polymorphism in the types of the latter two functions makes them suitable for probing other objects.

\subsection{Testing Properties at Quiescence}

In the previous section we have explained how properties of programs, processes, and concrete objects can be added and removed at run-time. In this section we show how these properties can be tested when the application is in a stable state, is quiescent. The function reportProperties evaluates all current properties of its program argument and collects the results in a report:

\footnotetext{
9 This is Clean's 'do-notation' for explicit environment passing.

$10+++$ is the Clean string concatenation operator.

11 dynamic $e:: t$ turns expression $e$ of type $t$ into a value of type Dynamic.
} 
reportProperties : : Program $\rightarrow$ PropertiesReport

The report assigns, for each step in a run, a verdict for each tested property. A verdict is a simple boolean, which is true iff the property holds.

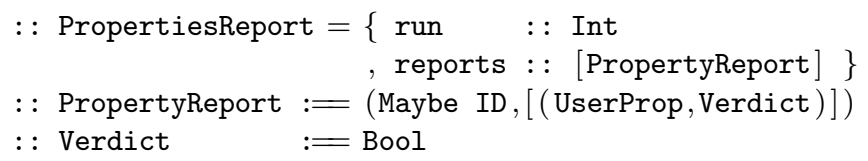

To keep track of the run, the program type is extended with a run-count that is incremented by eval:

$:$ Program $=\{\ldots$, run : : Int $\}$

The purpose of (reportProperties program) is to test every property in the props field of program. Recall that properties are boolean functions on the particular state of the object with which they are associated. This means that reportProperties must construct the appropriate state of each object at which the property function can be applied. The function can then compute the verdict simply by application of the property to the constructed state.

The top-level of this function is easily defined:

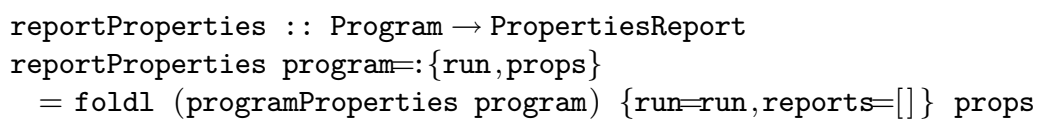

Note that this implies that if no properties are added to a program, the only computational overhead is generated by incrementing the run count by the modified eval function.

The function application (programProperties program pr prop) needs to test a program property in case prop $=$ (Nothing, props). We know that props contains (Prop [Process]) property functions because addProperty is type-safe.

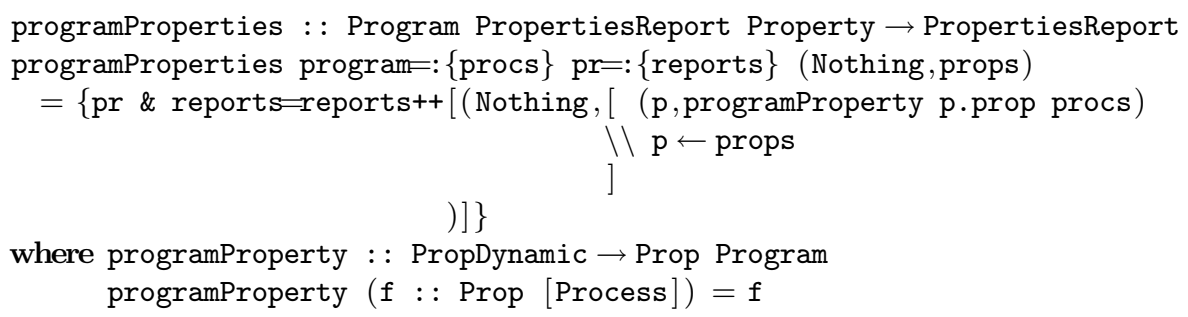

In case prop $=($ Just id,props $)$ then the correct state context needs to be built for a process or a concrete object. First consider testing a process property. If a process is found with a pid: :ID that matches id then we know that the list of properties props contains functions of type (Prop (Proc ps)), with ps the type of the current value of its state. We can safely unpack every such property using the dictionary of the process (pdict) and apply it to the process. Note that we need to filter itself from the list of all processes in the pcontext field before doing so. 


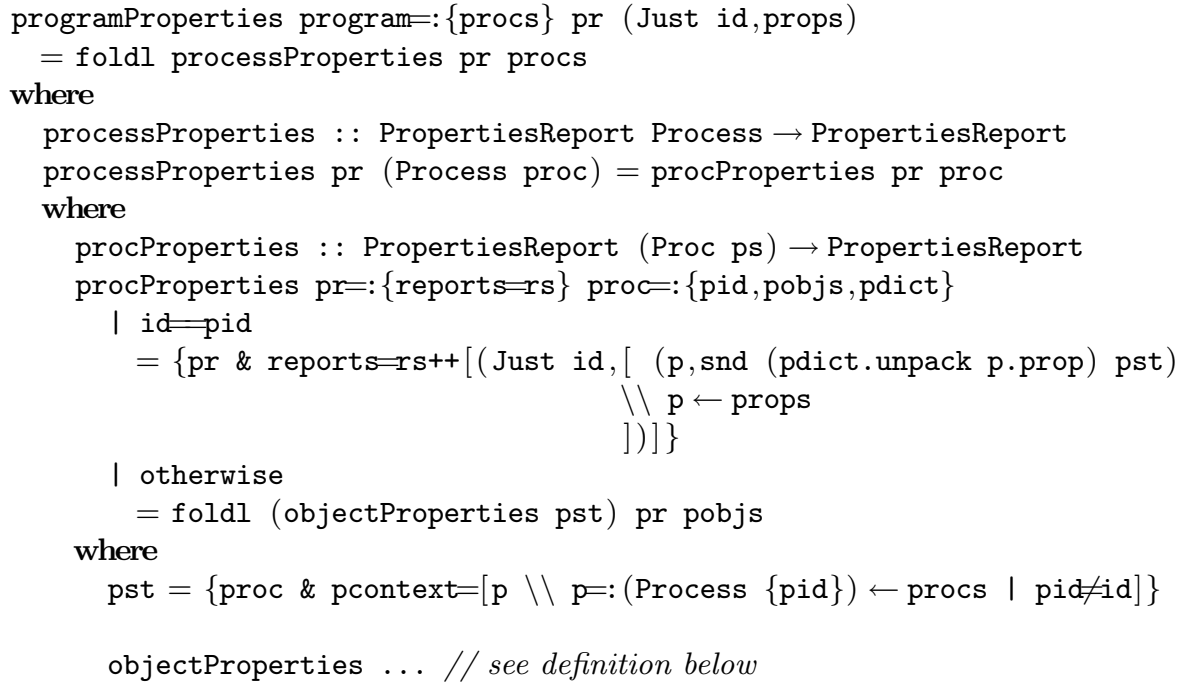

Testing concrete object properties proceeds in an analogous way, except that now a local state structure needs to be built to which the properties can be applied. This is a recursive definition that follows the nested structure of the objects and their state scopes, which makes it a bit verbose.

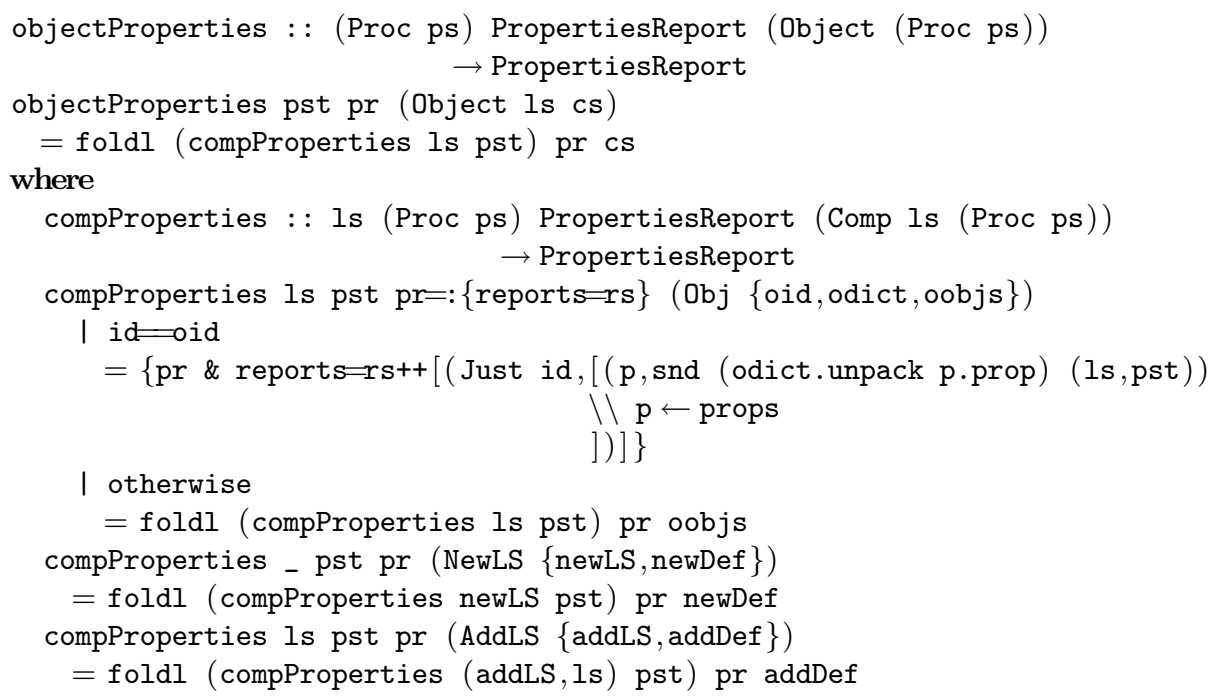

Given the reportProperties function, it is straightforward to define extended eval functions that produce property report(s) and next program(s):

step : : Event Program $\rightarrow$ (PropertiesReport,Program)

step event program $=$ (reportProperties program, eval event program)

steps :: [Event] Program $\rightarrow$ ([PropertiesReport], Program)

steps es program $=$ seqList (map step es) program 
Finally, for decent output, we define an instance of the toString function for a PropertiesReport that displays the number of tested properties, and names those that have failed in a particular run. We do not want to print the Program value, but we are interested in the definedness of its state values. We realize this by adding appropriate strictness annotations in its type definitions.

Example We finish our running example by mimicking a probing session of the faulty program. The program definition is extended with run=0 field. We start by running the program without any properties, given a particular scenario:

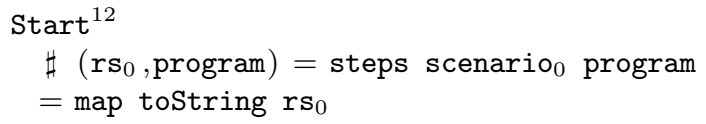

The scenario first picks the first action of concrete object with ID value equal to 2 , then takes the second action twice, and ends with the first action:

scenario $=[(2,1),(2,2),(2,2),(2,1)]$

This scenario reveals the first 'bug' in the program:

Step 0: tested 0 properties. 0 failing properties.

Step 1: tested 0 properties. 0 failing properties.

Step 2: tested 0 properties. 0 failing properties.

tl of []

This means that everything runs properly until just before the second invocation of the second action (step 2), but after doing that action apparently the tail of an empty list is taken. We want to probe the definedness of the integer list that can be accessed by concrete object identified by ID 2. For this purpose we use definedProp (Section 2.2).

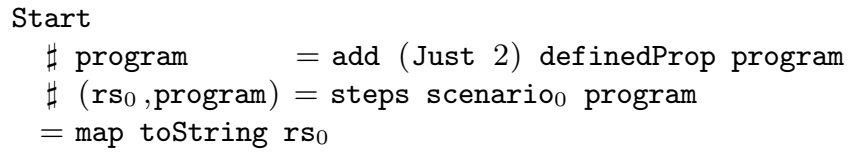

Applying the same scenario provides evidence that the list was defined initially and after the first two steps:

Step 0: tested 1 property. 0 failing properties.

Step 1: tested 1 property. 0 failing properties.

Step 2: tested 1 property. 0 failing properties.

tl of []

This bug is easily fixed by replacing tl with $t l^{\text {" }}$ :

$\mathrm{tl}^{6}::[\mathrm{a}] \rightarrow[\mathrm{a}]$

$\mathrm{tl}^{\prime} \mathrm{xs}=$ if (isEmpty $\left.\mathrm{xs}\right) \mathrm{xs}(\mathrm{tl} \mathrm{xs})$

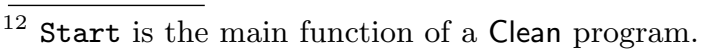


Running the program through the same scenario confirms that the definedness property now holds for this trace:

Step 0: tested 1 property. 0 failing properties.

Step 1: tested 1 property. 0 failing properties.

Step 2: tested 1 property. 0 failing properties.

Step 3: tested 1 property. 0 failing properties.

At this stage we no longer wish to probe the definedness of the list, but instead probe the other properties that have been given in Section 2.2. This is specified as follows:

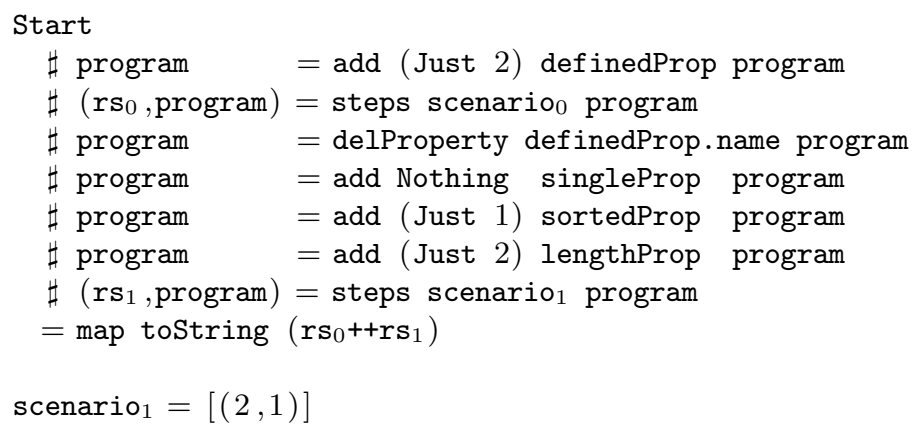

Running the program through this longer scenario reveals the second 'bug':

Step 0: tested 1 property. 0 failing properties.

Step 1: tested 1 property. 0 failing properties.

Step 2: tested 1 property. 0 failing properties.

Step 3: tested 1 property. 0 failing properties.

Step 4: tested 3 properties. 1 failing property.

lengthProp

Although property lengthProp is actually violated immediately after the second invocation of the second action, this is not displayed because the property was not probed at that stage. Instead, violation of the property is detected immediately after it is added (after step 3). This is caused by the local integer that decreases below 0 and therefore incorrectly reflects the length of the integer list. We fix this bug by replacing $\mathrm{n}-1$ with $\mathrm{n} .-.1$, defined as:

$(.-.)^{13}$ infixl $6:: !^{14}$ Int $!$ Int $\rightarrow$ Int

$(.-.) \mathrm{m} \mathrm{n}=\max 0(\mathrm{~m}-\mathrm{n})$

Running the scenario again renders the properties invariant with respect to this event trace.

$\overline{13(f) \text { fix } n::} t$ defines a new operator $f$ of type $t$ with fixity fix $\in\{$ infix, infixl, infixr $\}$ and precedence $n$.

${ }^{14}$ In a type definition, ! $t$ puts type $t$ in a strict context. 


\section{Object I/O is a Local State Transition Systems}

In this section we explain the relation between the local state transition systems of the previous section with Object I/O. We do this in an informal manner, by means of an Object I/O program that is equivalent to the one shown in Section 2. A different account has appeared earlier in [3].

As in the local state transition example, the program consists of a single interactive process. Instead of manipulating an integer list, this program manipulates an Id list (second argument of Process):

Start : : $*{ }^{15}$ World $\rightarrow *$ World

Start world $=$ startProcesses

[Process MDI [] initGUI [ProcessClose closeProcess]] world

In Object I/O the state of an interactive process is given by the record type $(::$ PSt ps $=\{1 s::$ ps, io : : IOSt ps $\}$ ), with ps the state as discussed in the previous section. io is a combination of the fields pobjs and pcontext. It is an abstract data type by which the programmer must access all GUI elements and the external world. In this example, we have a (PSt [Id]) process state.

The Id values are used to identify windows that are opened and closed dynamically by the two actions open and close. These actions are the callback functions of two menu items, labelled "Open" and "Close" respectively. Their parent object is the top-level menu object that corresponds with the Object of the local state transition system, and indeed, it encapsulates an integer state (first argument of openMenu). Note that the Object I/O program below has exactly the same 'bugs' as the running example in the previous section.

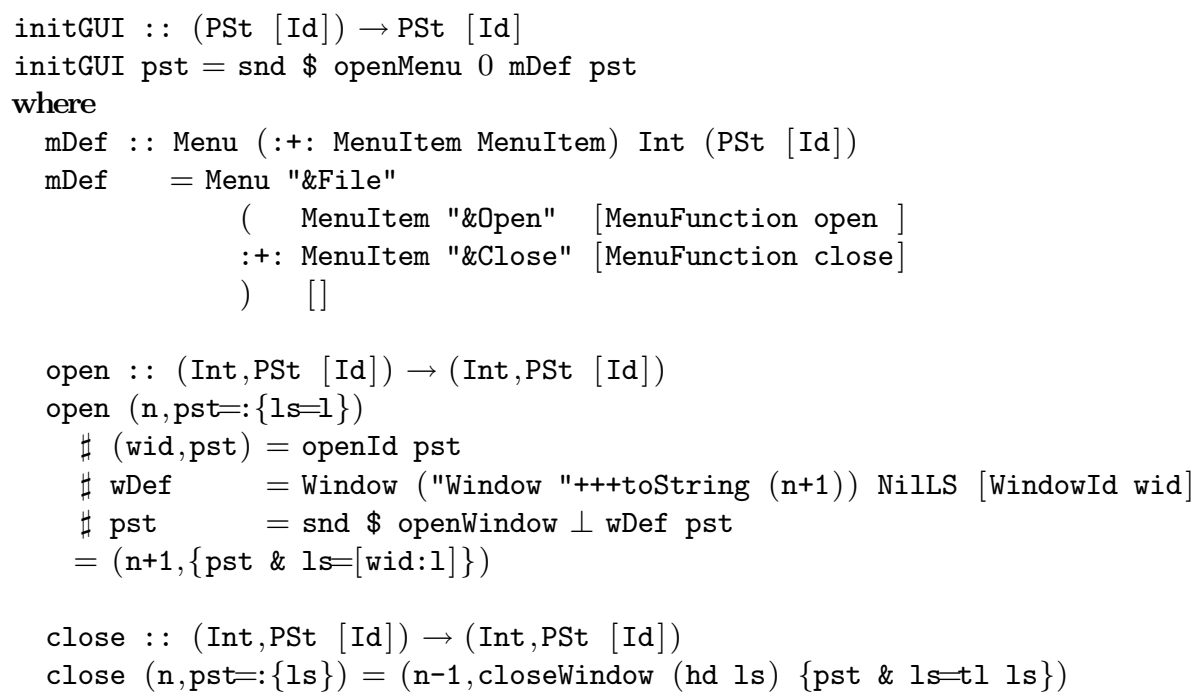

Recall that in the local state transition system there are three kinds of elements that can be probed:

$\overline{15}$ Clean uses the 'world-as-value' paradigm for I/O. An annotated type $* t$ indicates that $t$ is used in a single threaded way. This is guaranteed by the type system. 
Programs probed with (Prop [Process]). Object I/O has a data type similar to [Process] (viz. Context) but this is an internal data type and should not be accessed by the programmer. There are no retrieval operations defined on this data type, so basically, the programmer can not define program properties as in the previous section. (Note that this suggests that the API of Object I/O might be lacking functionality, so it is worthwhile to see what useful access functions can be added.)

Processes probed with (Prop (Proc ps)). Object I/O processes are probed by (Prop (PSt ps)); in the example by (Prop (PSt [Id])).

Objects probed with (Prop (1s,Proc ps)). The GUI objects in the program are mDef, its two MenuItem elements, and the dynamically created windows (wDef). The menu and its items share an integer local state, so they are probed by (Prop (Int,PSt $[\mathrm{Id}]))$. The window has no significant local state $(\perp:: \forall \mathrm{a}: \mathrm{a})$, so it is probed by $(\forall a$ :Prop (a,PSt [Id])).

\section{Issues of Probing Object I/O Programs}

In the previous section we have shown in a very informal way how Object I/O relates to local state transition systems. In this section we discuss the major issues that are likely to occur when Object I/O applications are dynamically probed. Firstly, dynamically adding/removing properties to an Object I/O program requires identification of the GUI elements at run-time (Section 4.1). Secondly, assertions should be free of side-effects (Section 4.2). Finally, this work contributes to an old debate about which state paradigm to use: explicit or implicit state passing (Section 4.3).

\subsection{Run-Time Identification of GUI elements}

From the account in Section 2.2 we know that it is sufficient to retrieve the Id value of an element in order to associate a property with it using addProperty. The example in Section 3 shows that these identification values are known only at run-time, which is a quite common approach in GUI APIs. When probing GUI elements dynamically, the user needs to identify them. For this purpose we include a GUI browser for each application with which the user can select a GUI element, and thereby its identification value. This browser can be defined in Object I/O using the API inspection functions, and a tree list control to present the hierarchical structure of the GUI. Fig. 1 gives screenshots of this browser for the example program at several stable states.

Clean dynamics can be stored on disk, so the user can browse the file system in search of interesting properties, or create them using the Clean IDE and store them on disk. This gives the two arguments of the addProperty function, which should allow us to associate a stored property with a given GUI element.

\subsection{Properties Should Have No Side-Effects}

A fundamental problem with assertion checking systems is the side-effect problem [10]. It states that properties should, for obvious reasons, have no side-effects on 

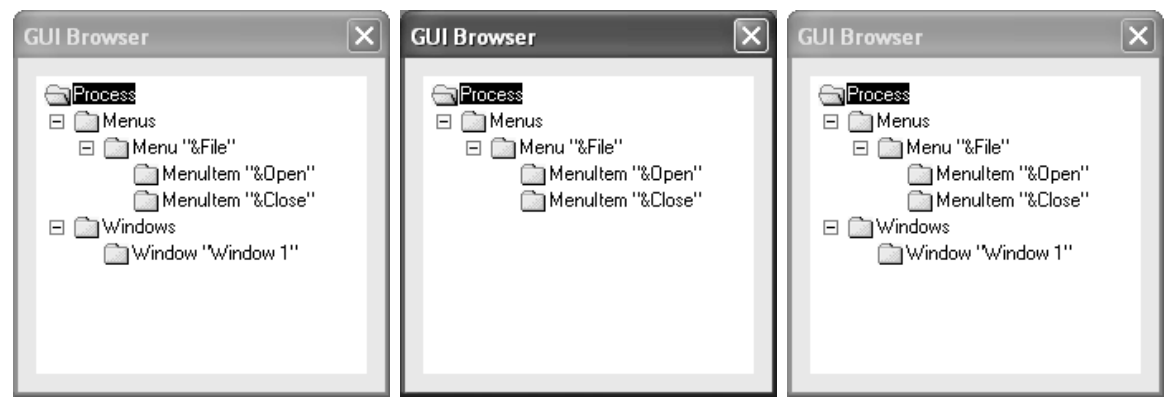

Fig. 1. The GUI browser after: $[(2,1)],[(2,1),(2,2),(2,2)]$, and $[(2,1),(2,2),(2,2),(2,1)]$.

the programs. In local state transition system terminology, this means that a property should not change the state that it inspects. In Section 2.2 this was effortlessly realized by defining a property over a state st as the simple function type $s t \rightarrow$ Bool. We would like to adopt this simple scheme to Object I/O, but unfortunately this is not possible. The main reason is that the types in Object I/O have been designed to allow the programmer to use unique state, i.e. state that can be destructively updated [6]. This requires the 'container types' to be at least as unique as their content. As a consequence, we are forced to use the following property type:

$:$ Prop st $:=$ st $\rightarrow($ Bool, st $)$

How can we guarantee that property functions have no side-effect? Such a function might have an effect on the custom states and on the GUI state. The latter can easily be eliminated by providing a 'mirror' library of Object I/O from which all functions are removed that have a side-effect. What is left is a proof obligation that property functions do not change the custom states. Systems with proof obligations require good support of proof tools such as Sparkle [9] in order to assist the programmer with these proofs.

\subsection{The Influence Of The State Paradigm}

Object I/O uses an explicit state passing paradigm. One advantage of this paradigm is that each object carries in its type full information about which state it manipulates, so we can quickly check if a property that is to be associated with an object actually matches the type of the state.

However, it is also possible to use an implicit state passing paradigm using MVars [13]. This has been discussed in [1]. The used GUI monad is a regular IOSt state monad which uses MVars to hold the logical state. Advantages of this approach are the simpler types of Object I/O GUI elements, and the ability to have more complex state structures without loss of control over access. The major disadvantage that was raised against its use is its less declarative nature because programmers need to explicitly take and put values from these variables. 
Interestingly, this paper identifies a new disadvantage of implicit state passing: in contrast with explicit state passing, the type of an object no longer contains information on the state that is manipulated by the object. This means that our approach of unifying the type of a dynamic property with the state of an object no longer works. Instead, one needs to associate properties over MVars that happen to be manipulated by objects. Identification and matching of MVars against property types can be done in a similar way as object identification in Sections 4.1 and 2.2, but is more complicated because all MVars must be retraceable for identification purposes, and a property of type $\left(\right.$ st $_{1} \ldots$ st $_{n} \rightarrow$ GUI Bool) must be matched against $\left(\right.$ MVar $\left.\mathbf{s t}_{1}\right) \ldots\left(\right.$ MVar $\left.\mathbf{s t}_{n}\right)$.

\section{Related Work}

Assertion checking has been integrated in the object-oriented languages Eiffel [12] and JML- Java Modelling Language [10]. The Objective Caml language [11] has an assert statement. In a recent experiment, assertion checking has been added to Haskell [7]. We have in common with the Eiffel approach that we want to use executable properties. With the JML approach we share the reuse of the host language and libraries in order to encourage programmers to probe their applications. The assert statement in Objective Caml evaluates boolean expressions. These have no effect in case of true statements, but an exception is thrown in case of false statements. The latter aspect is different from our approach: false properties increase ones understanding of an application as much as true properties, and therefore do not terminate the application.

The main differences are: because of the side-effect problem, JML can't handle I/O methods, which is clearly a must in our case; we do not annotate source code for our properties, but rather probe the application at run-time using dynamically associated properties; this requires properties to be persistent; we do not yet intend these properties to be subject to formal verification as in JML; in contrast with the Haskell approach in which properties are not asserted over unevaluated expressions, we think that an assertion should evaluate an unevaluated expression if needed. The reason for this is that our assertions are meant to express properties of a program: a list must be sorted, a length invariant should hold, and so on. Such properties do not stop at unevaluated expressions, but refer to the complete value.

Probing application properties dynamically has the same flavor as using a tracing/debugging tool such as Freja, Hat, and Hood [8] or those used in more conventional programming languages such as $\mathrm{C}$. With such tools one inspects the run-time values of an application whereas we focus on relations between run-time values expressed as properties.

Another area that is related to our work is that of testing [17] because in both areas it is the application itself that is subject to probing and we can give verdicts only for specific event traces, which in practice will not exhaust the possible event trace search space. At this moment the theory and practice of 
testing of GUI applications is starting to grow. Our project is a first step to investigate what can be done in this area.

\section{Conclusions and Future Work}

In this paper we have shown how systems that are based on local state transition systems can be probed at run-time for their stable state based properties. These properties can be added and removed at any stable state of the application. There are no limitations to the size of the application. We have shown what needs to be done additionally for one particular instance of local state transition systems, the Object I/O library. This provides us with a directly usable means to probe GUI applications of arbitrary size.

There are many directions of research to take based on this framework. Among others these are: adding good property management functionality to the framework; extend it with value-inspection and back-tracing in case a property is found to be invalid; explore the formal verification potential of our approach.

\section{Acknowledgements}

The author would like to thank Marko van Eekelen and the anonymous referees.

\section{References}

1. P. Achten and S. Peyton Jones. Porting the Clean Object I/O library to Haskell. In M. Mohnen and P. Koopman, editors, Proceedings of the 12th International Workshop on the Implementation of Functional Languages, IFL'00, Selected Papers, volume 2011 of $L N C S$, pages 194-213. Aachen, Germany, Springer, Sept. 2001.

2. P. Achten and R. Plasmeijer. Interactive Functional Objects in Clean. In C. Clack, K. Hammond, and T. Davie, editors, Proc. of the 9th International Workshop on the Implementation of Functional Languages, IFL 1997, Selected Papers, volume 1467 of $L N C S$, pages 304-321. St.Andrews, UK, Springer, Sept. 1998.

3. P. Achten and R. Plasmeijer. The implementation of interactive local state transition systems in Clean. In P. Koopman and C. Clack, editors, Proceedings of the 11th International workshop on the Implementation of Functional Languages, IFL'99, number LNCS 1868, pages 115-130. Springer-Verlag, Sept. 2000.

4. P. Achten and M. Wierich. A Tutorial to the Clean Object I/O Library - Version 1.2. Technical Report CSI-R0003, Computing Science Institute, Faculty of Mathematics and Informatics, University of Nijmegen, The Netherlands, Feb. 2000. 294 pages.

5. K. Angelov. ObjectIO for Haskell. Description and Sources at www.haskell.org/ObjectIO/, Applications at /free.top.bg/ka2_mail/, 2003.

6. E. Barendsen and S. Smetsers. Uniqueness typing for functional languages with graph rewriting semantics. In Mathematical Structures in Computer Science, volume 6, pages 579-612, 1996 . 
7. O. Chitil, D. McNeill, and C. Runciman. Lazy Assertions. In Greg Michaelson and Phil Trinder, editors, Draft Proceedings of the 15th International Workshop on the Implementation of Functional Languages, IFL'03, pages 31-46. Edinburgh, UK, Sept. 2003.

8. O. Chitil, C. Runciman, and M. Wallace. Freja, Hat and Hood - A Comparative Evaluation of Three Systems for Tracing and Debugging Lazy Functional Programs. In M. Mohnen and P. Koopman, editors, Proceedings of the 12th International Workshop on the Implementation of Functional Languages, IFL'00, Selected Papers, volume 2011 of LNCS, pages 176-193. Aachen, Germany, Springer, Sept. 2001.

9. M. de Mol, M. van Eekelen, and R. Plasmeijer. Theorem proving for functional programmers - Sparkle: A functional theorem prover. In T. Arts and M. Mohnen, editors, The 13th International Workshop on Implementation of Functional Languages, IFL 2001, Selected Papers, volume 2312 of LNCS, pages 55-72, Stockholm, Sweden, 2002. Springer.

10. G. T. Leavens, Y. Cheon, C. Clifton, C. Ruby, and D. R. Cok. How the Design of JML Accomodates Both Runtime Assertion Checking and Formal Verification. In Formal Methods for Components and Objects, volume 2852 of LNCS, pages 262-284. Springer Verlag, 2003. Also available as Technical Report TR 03-04a, Department of Computer Science, 226 Atanasoff Hall, Iowa State University, Ames, Iowa, USA.

11. X. Leroy. The Objective Caml system - release 3.08; Documentation and user's manual. Institut National de Recherche en Informatique et en Automatique, July 2004 .

12. B. Meyer. Eiffel: The Language. Prentice Hall, 1992.

13. S. Peyton Jones, A. Gordon, and S. Finne. Concurrent Haskell. In 23rd ACM Symposium on Principles of Programming Languages (POPL'96), pages 295-308, St.Petersburg Beach, Florida, 1996. ACM.

14. S. Peyton Jones and Hughes J. et al. Report on the programming language Haskell 98. University of Yale, 1999. http://www.haskell.org/definition/.

15. M. Pil. Dynamic types and type dependent functions. In D. Hammond and Clack, editors, Implementation of Functional Languages (IFL '98), LNCS, pages 169-185. Springer Verlag, 1999.

16. R. Plasmeijer and M. van Eekelen. Concurrent CLEAN Language Report (version 2.0), December 2001. http://www.cs.kun.nl/ clean/contents/contents.html.

17. J. Tretmans. Test Generation with Inputs, Outputs, and Quiescence. In T. Margaria and B. Steffen, editors, Second Int. Workshop on Tools and Algorithms for the Construction and Analysis of Systems (TACAS'96), volume 1055 of Lecture Notes in Computer Science, pages 127-146. Springer-Verlag, 1996.

18. M. Vervoort and R. Plasmeijer. Lazy dynamic input/output in the lazy functional language Clean. In R. Peña and T. Arts, editors, The 14th International Workshop on the Implementation of Functional Languages, IFL'02, Selected Papers, volume 2670 of $L N C S$, pages 101-117. Springer, Sept. 2003. 